\title{
SOME UNSOLVED PROBLEMS ON MEROMORPHIC FUNCTIONS OF UNIFORMLY BOUNDED CHARACTERISTIC
}

\author{
SHINJI YAMASHITA \\ Department of Mathematics \\ Tokyo Metropolitan University \\ Fukasawa, Setagaya, \\ Tokyo 158, Japan \\ (Received February 14, 1985)
}

ABSTRACT. The family $\operatorname{UBC}(R)$ of meromorphic functions of uniformly bounded characteristic in a Riemann surface $R$ is defined in terms of the Shimizu-Ahlfors characteristic function. There are some natural parallels between $U B C(R)$ and $B M O A(R)$, the family of holomorrhic functions of bounded mean oscillation in R. After a survey some open problems are proposed in contrast with $B M O A(R)$.

KEY WORDS AND PHRASES. Riemann surface, Shimizu-Ahlfors' characteristic function, $U B C(R), B M O A(R)$, 'rarmonic majorant, $F$. Riesz' decomposition, Carleson measure, classification of Riemann surfaces.

1980 MATHEMATICS SUBJECT CLASSIFICATION CODES. Primary 30D35, 30D45, 30D50, 30D55, 30F20. Secondary 30C8\%, 30C85, 30F30.

1. INTRODUCTION :

In a series if papers [19] $\sim$ [21], [23] [25] I have been studying functions of uniformly bounded characteristic. After a survey I propose some questions which I have been unable to answer. The adjective "unsolved" in the present title, therefore, means more precisely "unsolved by the present author".

Let $R$ be a Riemann surface which has the Green functions $g_{R}(z, w)$ with poles $w$ in $R$. As usual, each point of $R$ is identified with its local-parametric image in the complex plane. By $D$ we always mean a subdomain of $R$ such that the closure $D U \partial D$ is compact and the boundary $\partial D$ consists of a finite number of mutually disjoint, analytic, simple and closed curves in R. For a point $w$ of $D$ we set

$$
r=\exp \left\{\lim \left(g_{D}(z, w)+\log |z-w|\right)\right\}
$$

where $\mathrm{z} \rightarrow \mathrm{w}$ within the parametric disk of center $\mathrm{w}$.

Let $D_{t}=\left\{z \in D ; g_{D}(z, w) \geqq \log (r / t)\right\}, 0<t<r$. For $f$ meromorphic in $R$ we consider the second-order differential $f^{\#}(z)^{2} d x d y$, where $f^{\#}=\left|f^{\prime}\right| /\left(1+|f|^{2}\right)$. The Shimizu-Ahlfors characteristic function of $f$ is defined for a pair w, $D$ with $w \in D$ by

$$
T\left(D, w, f^{\cdot}\right)=\pi^{-1} \int_{0}^{r} t^{-1}\left[\iint_{D_{t}} f^{\#}(z)^{2} d x d y\right] d t .
$$


In the case $\mathrm{H}=\Delta \equiv\{|z|<1\}, \mathrm{w}=0, \mathrm{D}=\{|z|<r\}, 0<r<1$, wt, bt, in

$$
T(r, f)=T(D, w, f),
$$

the usual Shimizu-Ahlfors characteristic function of $f$. Returning to general $R$ we now set

$$
T(R, w, f)=\lim _{w \in D \uparrow R} T(D, w, f)
$$

this means that given $\varepsilon>0$ we may find a compact set $K \subset R$ ( $W \in K$ ) such that $|T(R)-T(D)|<\varepsilon$ for all $D \supset K$ with the obvious change in case $T(R)=\infty$. It is inown that

$$
T(R, w, f)=\pi^{-1} \iint_{R} f^{\#}(z)^{2} g_{R}(z, w) d x d y, \quad w \in R,
$$

.2 Grecn potential of the measure $\pi^{-1} f^{\#}(z)^{2} d x d y$ in $R$.

If $T(R, w, f)<\infty$ for a $w \in R$, then $T(R, w, f)<\infty$ for all $w \in R$. We call $f$ to be of bounded characteristic, $f \in B C \equiv B C(R)$ in notation, if $T(R, w, f)<\infty$ for a (hence for each) $w \in R$. Thus, $f \in B C$ if and only if $f$ is Lindelöfian and meromorphic in $R$ in the sense of Maurice Heins.

By definition, $f$ is of uniformly bounded characteristic, $f \in U B C \equiv U B C(R)$ in notation, if

$$
\mathrm{T}(\mathrm{R}, \mathrm{f}) \equiv \sup _{\mathrm{w} \in \mathrm{R}} \mathrm{T}(\mathrm{R}, \mathrm{w}, \mathrm{f})<\infty .
$$

Each meromorphic $f$ in $R$ can be expressed as a quotient $f=f_{1} / f_{2}$ of holomorphic functions $f_{1}$ and $f_{2}$ with no common zero in $R$. Therefore,

$$
\Phi=\log \left(\left|\mathrm{f}_{1}\right|^{2}+\left|\mathrm{f}_{2}\right|^{2}\right)
$$

is a finite-valued subharmonic function in $R$ because $\Delta \Phi(z) d x d y=4 f^{\#}(z)^{2} d x d y$. If $f \in B C$, then

$$
\phi_{R}^{\wedge}(w)-\Phi(w)=2 T(R, w, f), \quad w \in R,
$$

where $\Phi_{\mathrm{R}}^{\wedge}$ is the least harmonic majorant of $\Phi$ in $R$, the smallest among all the harmonic majorants of $\Phi$ in $R$. Therefore, the function $T(R, w, f)$ of $w$ is the potential part of the F. Riesz decomposition of $\Phi$ in $R$. Apparently, $f \in U B C$ if and only if $\Phi_{R}^{\wedge}$ exists (namely, $f \in B C$ ) and the potential part is bounded in $R$.

In the special case $R=\Delta$ we can choose as $D$ the non-Euclidean disks

$$
\Delta(w, r)=\{|z-w| /|1-\bar{w} z|<r\}
$$

of center $w \in \Delta$ and the radii $\tanh ^{-1} r, 0<r<1$, so that

$$
T(\Delta(w, r), w, f)=T\left(r, f_{w}\right),
$$

where $f_{w}(z)=f((z+w) /(1+\bar{w} z)), \quad z \in \Delta$.

It is not difficult to observe that $f \in \operatorname{UBC}(R) \Leftrightarrow f \circ p \in \operatorname{UBC}(\Delta)$, where $p: \Delta \rightarrow R$ is the universal covering projection. Actually, $T(R, p(a), f)=$ $T(\Delta, a, f \circ p)$. This fact reduces many problems on $\operatorname{UBC}(R)$ to those on $\operatorname{UBC}(\Delta)$, yet, in some cases, there are subtle differences; see the problem (I) below, for example. Hitherto we have been concerned with meromorphic functions $f$ in $R$. If $f$ is 
polc-:ree further. ther, we can propose wimilar considerations on reilacing $\mathrm{f}^{\#}$ by $\left|f^{\prime}\right|$. Thus, beginning with

$$
\mathrm{T} *(\mathrm{D}, \mathrm{w}, \mathrm{f})=\pi^{-1} \int_{0}^{\mathrm{r}} \mathrm{t}^{-1}\left[\iint_{D_{t}}\left|\mathrm{f}^{\prime}(\mathrm{z})\right|^{2} \mathrm{dxdy}\right] \mathrm{dt},
$$

we obtain $T^{*}(R, w, f)$ and others. If $T^{*}(R, w, f)<\infty$ for a $w \in R$, then $f \in H^{2}(R)$, that is, $|f|^{2}$ has the least harmonic majorant $\left(|f|^{2}\right)_{R}^{\wedge}$ in $R$, so that, $T^{*}(R, w, f)$ is finite for all $w \in R$. The converse is also true. To observe these we remember that if $f \in H^{2}(R)$, then

$$
\left(|f|^{2}\right)_{R}^{\wedge}(w)-|f|^{2}(w)=2 T *(R, w, f), \quad w \in R,
$$

an analogue of (1), holds [21], [23]. The left-hand side of (3) coincides with

$$
\left(|f-f(w)|^{2}\right)_{R}^{\wedge}(w), \quad w \in R .
$$

The obvious analogue of (2), together with (3), yields in $\Delta$ the identity

$$
\left(\left|f_{w}-f(w)\right|^{2}\right)_{\Delta}^{\wedge}(0)=2 T^{*}\left(\Delta, f_{w}\right) \text {. }
$$

A holomorphic function $f$ in $R$ is called BMOA, $f \subseteq B M O A \equiv B M O A(R)$ in notation, if

$$
\mathrm{T} *(\mathrm{R}, \mathrm{f}) \equiv \sup _{\mathrm{w} \in \mathrm{R}} \mathrm{T} *(\mathrm{R}, \mathrm{w}, \mathrm{f})<\infty ;
$$

the notion of $B M O A(R)$ was first introduced by Thomas A. Metzger, and the present author extended it to many-valued functions [23]. In case $R=\Delta$, this coincides with the known class $B M O A(\Delta)$. The inclusion formula $B M O A(R) \subset \operatorname{UBCA}(R)$ is obvious, where $\operatorname{UBCA}(R)$ is the family of all the holomorphic functions in $U B C(R)$. This is a consequence of the obvious inequality $T(R, f) \leqq T^{*}(R, f)$, yet a better estimate

$$
T(R, f) \leqq 2^{-1} \log \left\{2 T^{*}(R, f)+1\right\}
$$

can be proved. There exists $f \in \operatorname{UBCA}(\Delta) \backslash \operatorname{BMOA}(\Delta)$.

It is known that if $f \in \operatorname{BMOA}(\Delta)$, then $f$ is Bloch, $f \in B(\Delta)$ in notation, in the sense that

$$
\sup _{z \in \Delta}\left(1-|z|^{2}\right)\left|f^{\prime}(z)\right|<\infty \text {. }
$$

Similarly, it is known [19] that if $f \in \operatorname{UBC}(\Delta)$, then $f$ is normal in the sense of $011 i$ Lehto and Kaarlo I. Virtanen, $f \in N(\Delta)$ in notation, that is,

$$
\sup _{z \in \Delta}\left(1-|z|^{2}\right) f^{\#}(z)<\infty \text {. }
$$

Both $B(\Delta)$ and $N(\Delta)$ can be extended to $B(R)$ and $N(R)$ because $R$ has the hyperbolic metric. The inclusion formulae $B M O A(R) \subset B(R)$ and $U B C(R) \subset N(R)$ are proper in the case $R=\Delta$. (See the papers [1] [18], [22], [25], on normal meromorphic functions and the related topics.) Algebraically, $B M O A(R)$ is closed for summation, while $U B C(R)$ is not; UBC resembles $N$ at this point.

\section{PROBLEMS :}

Now, the problems:

(I) For $\alpha \in \mathbb{C}^{*} \equiv\{|z| \leqq \infty\}$ we let $n(\alpha, f)$ be the number of the roots of the equation $f=\alpha$ in $R$. Suppose that there exists an integer $k \geqq 0$ such that

$$
\operatorname{cap}\left\{\alpha \in \mathbb{C}^{*} ; \mathrm{n}(\alpha, \mathrm{f}) \leqq \mathrm{k}\right\}>0,
$$


valid for $R$ in case $k=0$, and is valid for $R=\Delta$ and $k \geqq 0$. The fut. em is unsolved for $k>0$ on general $R$. Earlitr and well-known conclusion is that $f \in$ $\mathrm{BC}(\mathrm{R})$.

(II) Let $A(R, f)$ be the spherical area of the image $f(R)$ of $R$, that is, the projection to $\mathbb{C}^{*}$ of the Riemannian image of $\mathrm{R}$ by $\mathrm{f}$. Is there a constant $\mathrm{k}>0$ sursh that

$$
T(R, f) \leqq k A(R, f) ?
$$

Since $A(R, f) \leqq \pi$ always holds if the sphere $\mathbb{C}^{*}$ is considered to have the radius $1 / 2$, the answer is "no" in general. We must therefore ald the condition that $A(R, f)$ $<\mathrm{T}$; in this case $\mathrm{T}(\mathrm{R}, \mathrm{f})<\infty$ is obvious; see ( $\mathrm{I})$. The celebrated Herbert $\mathrm{J}$. Alexander-B. A. Taylor-Joseph L. Ullman inequality teaches us that for holomorphic $f$,

$$
T^{*}(R, f) \leqq(2 \pi)^{-1} A^{*}(R, f),
$$

where, in this case, $A^{*}(R, f)$ is the Euclidean area of $f(R)$. See the problem (VII) below.

(III) As usual, let $O_{X}$ be the family of open Riemann surfaces $R$ of class $O_{G}$ or of those which are hyperbolic with $X(R)=\mathbb{C}^{*} \backslash\{\infty\}$. It is easy to observe that

$$
\mathrm{O}_{\mathrm{UBCA}} \subset \mathrm{O}_{\mathrm{BMOA}}
$$

To prove that the inclusion is proper we make a few modifications to the argument in [23]. Let $E$ be a compact set of linear measure zero, yet of positive capacity, lying on the real axis. Let $a \in E$ and let $h(z)=1 /(z-a), z \in \mathbb{C}^{*}$. We show that $R=\mathbb{C}^{*}-\mathrm{h}(\mathrm{E})$ is of $\mathrm{O}_{\mathrm{H}}{ }^{2}$ (hence of $\mathrm{O}_{\mathrm{BMOA}}$ ), yet $\mathrm{R} \notin \mathrm{O}_{\mathrm{UBCA}} \cdot$ First, the function $z$ is of $\operatorname{UBCA}(R)$ because it omits the set $h(E)$ of positive capacity, so that $R$ \& $O_{U B C A}$. Next, let $f \in H^{2}(R)$. Then foh $\in H^{2}\left(\mathbb{C}^{*}-E\right)$. Since $E$ is removable for $\mathrm{H}^{2}$, foh, and hence $f$ must be a constant. Therefore, $\mathrm{R} \in \mathrm{O}_{\mathrm{H}}{ }^{2}$. The problem is to

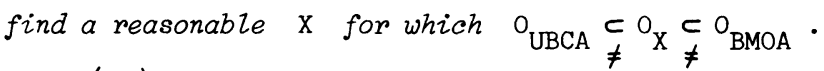

(IV) If $f \in \operatorname{UBC}(R)$, then $f \in \operatorname{UBC}\left(R_{1}\right)$ for each subdomain $R_{1}$ of $R$. Consider the converse in the special case $R=\Delta$. Let $\delta(w, r)=\Delta \cap\{|z-w|<r\}, w \in \partial \Delta$, $0<r<1$. Let $f$ be meromorphic in $\Delta$ such that for each $w \in \partial \Delta$ we may choose $0<r<1$ such that $f \in \operatorname{UBC}(\delta(w, r))$. Is it true that $f \in \operatorname{UBC}(\Delta)$ ? The corresponding problem for $B M O A$ is solved in the positive in [21]; the extensions to the bordered Riemann surfaces under the obvious technical conditions are now easy.

(V) Each $f \in U B C(\Delta)$ has, as a member of $B C(\Delta)$, the expression: $f=\left(B_{1} / B_{2}\right) F$, where $B_{1}$ and $B_{2}$ are Blaschke products with no common zero and $F$ is holomorphic and zero-free in $\Delta$. We observe that $F \in \operatorname{UBC}(\Delta)$. Conversely suppose that $F \in \operatorname{UBC}(\Delta)$ for $f \in B C(\Delta)$. Is it true that $f \in \operatorname{UBC}(\Delta)$ ? The answer is "no" [19]. Actually, there exists a nonnormal Blaschke quotient $B_{1} / B_{2}$; we have only to let $F=1$. What is a reasonable condition for $\mathrm{B}_{1} / \mathrm{B}_{2} \in \mathrm{UBC}(\Delta)^{2}$ ? An attempt is proposed in [25] in terms of uniformly separated sequences.

(VI) For $w \in W$ we set

$$
\begin{aligned}
\Sigma(w) & =\{z \in \Delta ;|w| \leq|z|<1,|\arg (z / w)|<\pi(1-|w|)\}, & & \text { if } w \neq 0 ; \\
& =\Delta, & & \text { if } w=0 .
\end{aligned}
$$


The length of the arc $\partial \Delta \cap \partial \Sigma(w)$ is $\ell(w)=2 \pi(1-|w|)$. A measure $\mu \geq 0$ in $\Delta$ is called a Carleson measure if $\sup \mu(\Sigma(w)) / \ell(w)<\infty$, where $w$ ranges over $\Delta$. It is known that $f \in \operatorname{BMOA}(\Delta)$ if and only if $\left(1-|z|^{2}\right)\left|f^{\prime}(z)\right|^{2} \mathrm{dxdy}$ is a Carleson measure (in the differential form). I proved [24] that if $f \in U B C(\Delta)$, then

$$
d \mu_{f}(z)=\left(1-|z|^{2}\right) f^{\#}(z)^{2} d x d y
$$

is a Carleson measure, and gave a partial answer for the converse. The problem is: $d \mu_{f} i s$ a Carleson measure. $\Rightarrow f \in \operatorname{UBC}(\Delta)$ ? I note that a difficulty lies in the fact that $\mathrm{f}^{\# 2}$ is not subharmonic in general.

(VII) Let $\mathrm{UBC}_{0}(R)$ be the family of meromorphic functions $f$ in $R$ such that $T(R, w, f) \rightarrow 0$ as $w \rightarrow \partial R$, namely, given $\varepsilon>0$ we may find a compact $K \subset R$ such that $T(R, w, f)<\varepsilon$ for $w \in R \backslash K$; this can be read in case $R=\Delta, T\left(1, f_{w}\right) \rightarrow 0$ as $|w|+1$; the holomorphic analogue is $V M O A(R)$ obtained on replacing $T$ by $T *$ in the above. It is not difficult to prove that $\mathrm{UBC}_{0}(R) \subset \mathrm{UBC}(R)$ and $\operatorname{VMOA}(R) \subset$ $B M O A(R)$. Furthermore, we see

$$
\text { fop } \in \mathrm{UBC}_{0}(\Delta) \Rightarrow f \in \mathrm{UBC}_{0}(R) \text {; and } f \circ p \in \operatorname{VMOA}(\Delta) \Rightarrow f \in \operatorname{VMOA}(R) \text {, }
$$

because the projection of a closed disk in $\Delta$ is compact in $R$. Are the converses valid? We remark that the following are known:

$$
\iint_{\Delta^{f^{\prime}}}(z)^{2} d x d y<\infty . \Rightarrow f \in \operatorname{UBC}_{0}(\Delta) ; \text { and } \iint_{\Delta}\left|f^{\prime}(z)\right|^{2} d x d y<\infty . \Rightarrow f \in \operatorname{VMOA}(\Delta) \text {; }
$$

see [19] and [24]. Are the extensions true for R? Metzger, in a communication, informed me some partial answers on the VMOA part. I do not know further information.

Finally we note that the hyperbolic analogue of $\operatorname{UBC}(R)$ is possible; see [21], [26], and a forthcoming paper [27]. Let $f$ be holomorphic and bounded, $|f|<1$, in $\mathrm{R}$, and let $\Psi=-\log \left(1-|f|^{2}\right)$. Then $\Psi$ is subharmonic with $\Delta \Psi(z) \mathrm{dxdy}=$ $4\left|f^{\prime}(z)\right|^{2} /\left(1-|f(z)|^{2}\right)^{2} d x d y$. The analogue of (1), and others, are true on replacing $\mathrm{f}^{\#}$ by the hyperbolic derivative $\left|f^{\prime}\right| /\left(1-|f|^{2}\right)$. We note that $\sigma(f, 0)=\tanh ^{-1}|f|$ is the non-Euclidean hyperbolic distance of $f$ and 0 , and $\sigma(f, 0)$ is subharmonic with $\Psi \leqq 2 \sigma(f, 0) \leqq \Psi+\log 4$. It is a future task to find some problems on this family.

The present article depends on the lecture on February 4, 1985, at Mie University, Tsu, Mie, Japan, where slightly abstract treatment beginning with the F. Riesz decomposition of subharmonic function was discussed.

\section{REFERENCES}

YAMASHITA, S. :

1. On K. Yosida's class (A) of meromorphic functions, Proc. Japan Acad. 50(1974), 347-349.

2. The derivative of a holomorphic function in the disk, Michigan Math. J. 21 (1974), 129-132

3. On normal meromorphic functions, Math. Zeits. 141(1975), 139-145.

4. Almost locally univalent functions. Monatsh. Math. 81(1976), 235-240.

5. Lectures on Locally Schlicht Functions, Tokyo Metropolitan University Mathematical Seminar Reports, iii+112 pages, 1977; sold at the library of the Department of Mathematics.

6. Cluster sets of set-mappings, Ann. Polon. Math. 35(1977), 77-98. 
7. Conformality and semiconformality of a function holomorphic in the disk, Trans. Amer. Math. Soc. 245(1978), 119-138.

8. The normality of the logarithmic derivative, Comm. Math. Univ. St. Pauli $27(1978)$, 25-28.

9. A non-normal function whose derivative has finite area integral of order $0<\mathrm{p} \&$, Ann. Acad. Sci. Fenn. Ser. A. I. Math. 4 (1978/1979), 293-298.

10. A non-normal function whose derivative is of Hardy class $H^{p}, 0<p<1$, Canad. Math. Bull. 23 $(1980)$, 499-500.

11. The normality of the derivative, Revue Roum. Vath. Pure Appl. 25(1980), 481-484.

12. Criteria for functions to be Bloch, Bull. Austral. Math. Soc. 21(1980), $223-227$.

13. Nonnormal Dirichlet quotients and nonnormal Blaschke quotients, Proc. Amer. Math. Soc. 80 (1980), 604-606.

14. Gap series and $\alpha-B l o c h$ functions, Yokohama Math. J. 28 (1980), 31-36.

15. The order of normality and meromorphic univalent functions, Colloguium Hath. 44 (1981), 159-163.

16. Bi-Fatou points of a Blaschke quotient, Math. Zeits. 176(1981), 375-377.

17. Non-normal Blaschke quotients, Pacific J. Math. 101(1982), 247-254.

13. Hardy norm, Bergman norm, and univalency, Ann. Polon. Math. 43(1983), 23-33.

1\%. Functions of uniformly bounded characteristir, Ann. Acad. Sci. Fenn. Ser. A. I. Math. I (1982), 349-367.

20. Exceptional sets, subordination, and fur' ti, of uniformly bounded characteris Math. Scand. 54 (1984), 242-252.

$2^{7}$. F. Riesz's decomposition of a subharmonic function, applied to BMOA, Boll. Un. Mat, . Ital. 3-A (1984), 103-109.

2\%. Area criteria for functions to be Bloch, normil, and Yosida, Proc. Japan Acad. 59 (1983), $462-464$.

$2 \%$ Furctions of uniformly bounded characteristic on Riemann surfaces, Trans. Amcr. Mat, h. Soc. 240 (1985), to appear.

24. Image area and functions of uniformly bounded characteristic, (omm. Math. Univ. St. Pauli, to appear.

2). Criteria for a Blaschke quotient to be of unitormly bounded characteristic, Iroc. Amer. Math. Soc., to appear.

26. Tout ensemble $\mathrm{H}^{2}$-enlevable est BMOA-enlevable, Comptes Rend. Acad. Sci. Pari:; 298 (1984), 509-511.

27. Holomorphic functiors of hyperbolically bounded mean oscillation, in preparation. 


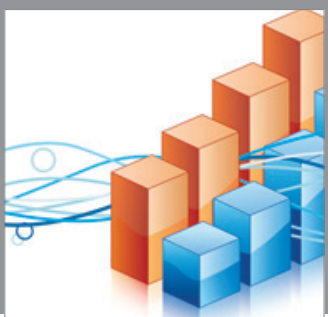

Advances in

Operations Research

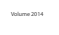

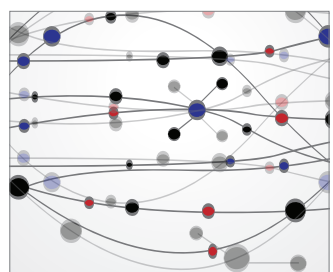

\section{The Scientific} World Journal
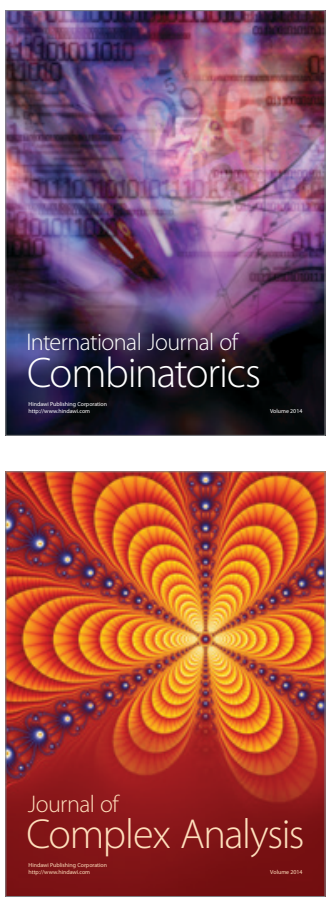

International Journal of

Mathematics and

Mathematical

Sciences
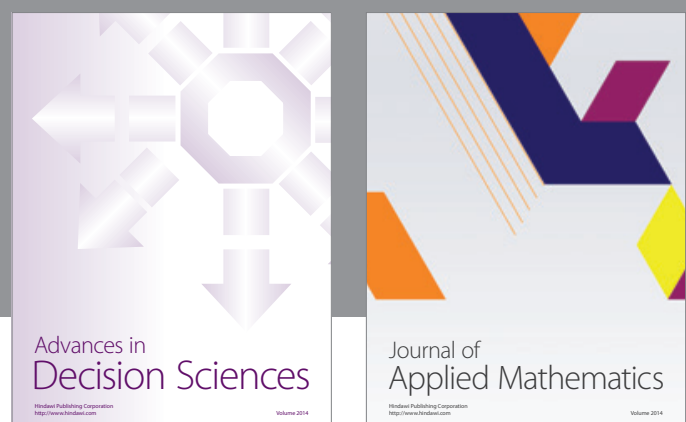

Journal of

Applied Mathematics
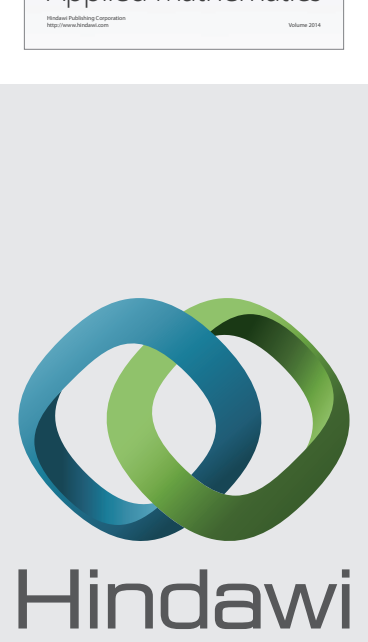

Submit your manuscripts at http://www.hindawi.com
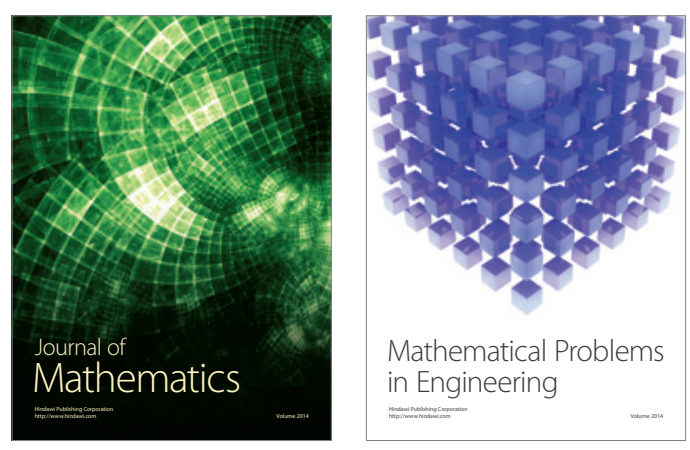

Mathematical Problems in Engineering
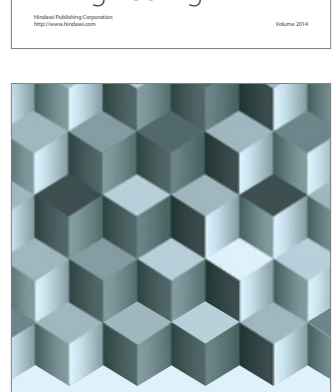

Journal of

Function Spaces
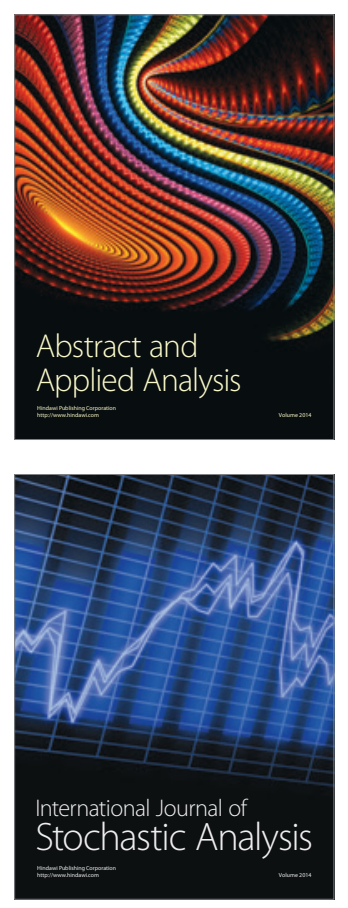

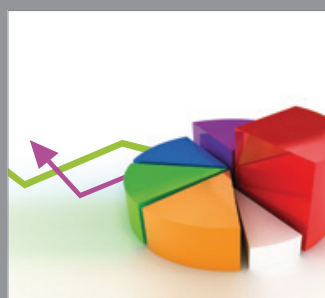

ournal of

Probability and Statistics

Promensencen
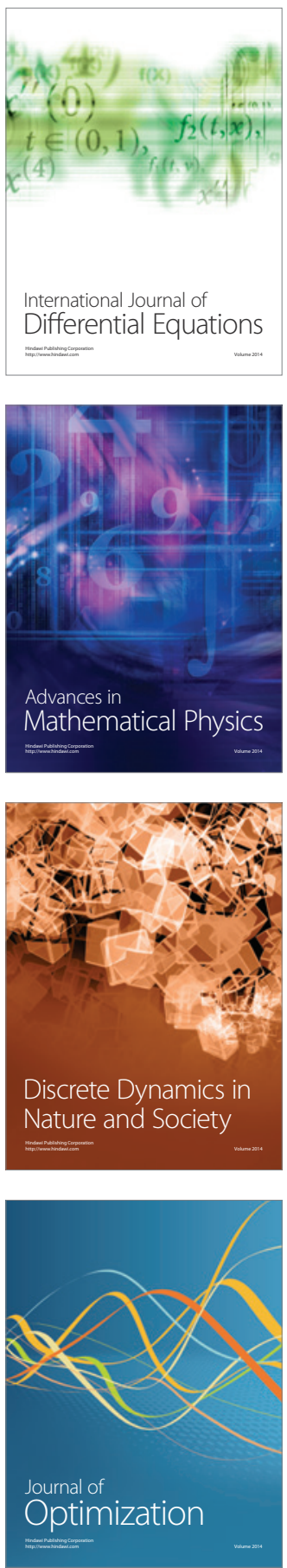